\title{
UNE VERSION CHINOISE DU CONTE BOUDDHIQUE DE KALYÂNAMKARA ET PÂPAMKKARA
}

PAR

\section{EDOUARD CHAVANNES.}

Au nombre des manuscrits rapportés de Touen-houang par Mi. Pelliot et déposés par lui à la Bibliothèque nationale se trouve un texte ouigour dans lequel on a reconnu le conte bouddhique des deux frères Kalyânạmkara et Pâpaṃkara; M. Cl. Huart a publié un déchiffrement et une traduction de ce manuscrit ${ }^{1}$ ); ce double travail a été complété sur plusieurs points par M. Pelliot ici-même ${ }^{2}$ ).

Comme l'ont remarqué MM. Huart et Pelliot, ce conte nous est déjà familier tant par des versions tibétaines que par des versions chinoises; aucune d'elles cependant ne présente un parallélisme rigoureux avec le texte turc. Bien plus étroitement apparentée à ce dernier est la rédaction chinoise qu'on va lire; les deux récits suivent une marche semblable et on peut, grâce à ce terme de comparaison, voir en quoi consistent les lacunes du manuscrit turc. Cependant l'identité n'est pas rigoureuse et il est évident que la version ouigoure ne doit pas être considérée comme la traduction de ce texte chinois.

1) Le conte bouddhique des deux frères en langue turque et en caractères oügours, par Cl. Huart (Journal asiatique, Janv.-Fév. 1914, p. 5-57).

2) La version oügoure de l'histoire des princes Kalyānamkara et Päpamkara par Paul Pelliot (Troung pao, Mai 1914, p. 225-272). 
Le sûtra chinois qui nous fournit ce conte nous en donne la plus ancienne rédaction; en effet, quoique le nom du traducteur soit ignoré, c'est à l'époque des Han postérieurs qu'est rapporté le Ta fang pien fo pao ngen king 大方便佛報恩經 où notre conte occupe les pages $15 \mathrm{~b}$ à 19 a dans l'édition du Tripițaka de T'ôkyô (vol. VI, fasc. 5).

Enfin, il est à remarquer que, dans l'iconographie de Touenhouang qui, selon toute vraisemblance est à peu près de la même époque que le manuscrit ouigour rapporté par M. Pelliot, le conte de Kalyânaṃkara et Pâpaṃkara est connu sous la forme que lui avait donnée le traducteur du Fo pao ngen king; en voici la preuve: les noms des princes Kalyânamkara et Pâpamkara sont rendus par les termes 善事 et 惡事 dans le Hien yu king et par les termes 善行 et 惡行 dans le Vinaya des Mûlasarvâstivâdin; dans le Ta fang pien fo pao ngen king, qui est plus ancien, les noms sont

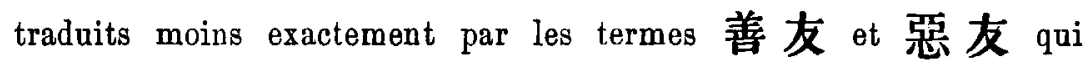
signifient, non pas "bonne action” et „mauvaise action”, mais „bon ami" et "mauvais ami". Or c'est précisément le terme 善友 que nous voyons apparaître dans une peinture de Touen-houang ') où la légende explicative s'inspire manifestement de la rédaction du Ta fang pien fo pao ngen king; enfin, M. Pelliot rappelle ${ }^{2}$ ) que, sur la paroi d'une des grottes de Touen-houang, on voit un bøuf qui lèche les yeux du prince 思友; il faut sans doute lire 善友, et c'est certainement un épisode de l'histoire de Kalyânạmkara qui était ici représenté.

En conclusion, les raisons qui nous ont déterminé à publier la

1) Cette peinture est une de celles qui ont été rapportées par M. Stein; je dois à l'obligeance de M. Petrueci la connaissance des légendes chinoises qui l'accompagnent.

2) Cf. T'oung pao, 1914, p. 228, 1. 10-13. 
conte bouddhique de Kalyânạnạkara et Pâpaṃkara.

traduction qu'on va lire sont les suivantes: $1^{\circ}$ ce texte est parallèle à celui du manuscrit ouigour de M. Pelliot; $2^{\circ}$ il est la plus ancienne rédaction d'un conte important; $3^{\circ}$ il éclaire deux des monuments iconographiques de Touen-houang.

Extrait du Ta fang pien fo pao ngen king (Tripitaka de Tókyô, vol. VI, fasc. 5, p. 15 b-19a).

Le Buddha dit: Aux temps passés, il y a de cela des milliers innombrables d'années, il $\mathrm{y}$ avait un royaume nommé Po-lo-nai (Vârânạasî, Bénarès); là était apparu dans le monde un Buddha appelé $\mathrm{P}^{c} \mathrm{i}$-po-che (Vipaçyin) jou-lai (tathâgata), ying-kong (méritant les offrandes = arhat), tcheng-pien (exact et complet = samyaksambuddha), tche-ming-hing-tsou (doué de la pratique de la science $=$ vidyâčaraṇasampanna), chan-tche (bien parti = sugata), che-kien-kiai (qui connaît le monde $=$ lokavit), wou-chang (sans supérieur $=$ anuttara), che-t'iao-yu-tchang-fou (cocher du domptable homme $=$ puruşadamyasârathi), t'ien-jen-che (maître des devas et des hommes $=$ çâstâ [maître d'enseignement]), fo (buddha = jina [vainqueur]), che-tsouen (honoré du monde $=$ lokajyeștha [aîné du monde]) ${ }^{1}$ ).

Etant présent dans le monde, il donna ses enseignements pendant une durée de dix fois mille années; après son nirvânạ, sa loi correcte (saddharma) demeura dans le monde pendant douze mille an-

1) La série d'épithètes que nous avons ici se retrouve an début de plusieurs des contes incorporés dans le Ta fang pien fo pao ngen ǩing; elle correspond, comme a bien voula me l'indiquer M. Sylvain Lévi, à une liste indienne qui apparait notamment en tête de la Mahâvyutpatti; en chinois, elle comporte les termes suivants qui accompagnent le nom de P'i-po-che 毗婆户 (Vipagyin): 如來。應供。正遍。知明行 足。善逝。世間解。無上。士調御丈夫。天人師。 佛。世尊。 
nées; après que sa loi contrefaite (pratirûpaka) ${ }^{1}$ ) se fut éteinte, il y eut un roi de Po-lo nai (Vârânasî, Bénarès), nommé Mo-ho-lo-chö 摩訶羅闃 (Mahârâja) ${ }^{2}$ ); il était intelligent et bon; il gouvernait son royaume suivant la loi correcte; il ne traitait pas injustement le peuple; ce roi dominait sur soixante petits royaumes et sur huit cents bourgs; il avait cinq cents éléphants blancs et vingt mille épouses, mais il n'avait aucun fils. Le roi en personne adressa des prières et des sacrifices aux divinités des montagnes, des fleures, des étangs et des arbres; après que douze années se furent écoulées, l'épouse que le roi honorait le plus se trouva enceinte; la seconde épouse elle aussi se trouva enceinte simultanément; le roi en fut très joyeux; de ses propres mains il faisait des offrandes (à ces deux femmes); pour leur couche, leur boire et leur manger il leur fii donner ce qu'il y avait de plus délicat. Lorsque les dix mois furent accomplis, le prince héritier naquit; son extérieur était bean; sun aspect merveilleux était élégant; les caractéristiques de l'homme étaient en lui au complet. La seconde épouse elle aussi mit simultanément au monde un fils. Le roi en fut très joyeux; il convoqua ses ministres, les fonctionnaires de tout rang et lez brahmanes maîtres dans l'art de la divination pour qu'ils tirassent l'horoscope (des enfants). Prenant dans ses bras le fils (de la première épouse), il le

1) Pour chaque Buddha qui apparaît dans le monde, on reconnait, après son Nirvaña qui clôt l'époque où il a enseigné lui-même, une période dite de la loi correcte 正法 pendant laquelle sa doctrine se maintient dans son intégrité, puis une période dite de la loi contrefaite 像法 pendant laquelle la religion n'est plus qu'un lointain reflet de ce qu'elle était au début. Tel est le vrai sens de cette expression 像法 qui a été souvent traduite par erreur comme signifiant la Loi des images.

2) Mahârâja n'est jas un nom propre; c'est un simple titre. Dans la version ouigoure, le père des deux princes est appelá Makhayt (cf. T'oung pao, Mai 1914, p. 232, n. 3); il est vraisemblable que ce terme est une déformation du titre de Mabârâja pris pour un nom propre. 
conte bouddhique de Kalyânạạ̣kara et Pâpaṃkara.

leur montra pour leur permettre de lui donner un nom; les maîtres dans l'art de la divination demandèrent: „A la naissance de cet enfant, quels pronostics heureux se sont produits?" On leur répondit: „Pour ce qui est du premier prince-héritier, sa mère avait un naturel primitivement mauvais; elle était méchante et jalouse; elle était arrogante et orgueilleuse; à partir du moment où elle fut enceinte de cet enfant, son caractère devint harmonieux et excellent; elle eut un visage affable et un air agréable; quand elle parlait, c'était en riant; sa première pensée était de s'informer des autres; ses doux propos faisaient du bien; elle était bonne et compatissante envers la multitude des êtres vivants qu'elle traitait comme s'ils eussent été de tout jeunes enfants". Les maîtres dans l'art de la divination répondirent: „C'est là un effet de la vertu qui porte bonheur de ce fils, car c'est grâce à lui que sa mère est devenue telle". Ils lui donnèrent donc le nom de prince Chan-yeou (excellent ami).

Quant au prince qu'avait enfanté la seconde épouse, les maîtres dans l'art de la divination demandèrent: „A la naissance de cet enfaut, quels pronostics heureux se sont produits?" On leur répondit: „Sa mère avait un naturel primitivement harmonieux et excellent; sa première pensée était de s'informer des autres; les paroles qu'elle prononçait étaient douces et pouvaient toucher le cœur de la multitude; à partir du moment où elle fut enceinte, son caractère devint soudain méchant; les paroles qu'elle prononçait étaient rudes et mauvaises; elle était jalouse et haineuse". Les maîtres dans l'art de la divination répondirent: „C'est là un effet des actes accomplis par cet enfant, car c'est grâce à lui que sa mère est devenue telle. Il faut donc lui donner le nom de Ngo-yeou (méchant ami)".

Allaités et nourris à la becquée, les enfants grandirent et attei- 
gnirent leur quatorzième année. Le prince Chan-yeou était intelligent et affectueux; il so plaisait à répandre des libéralités. Son père et sa mère avaient pour lui une affection toute particulière et le considéraient comme leurs oreilles ou leurs yeux. Quant au prince Ngoyeou, son caractère était méchant; son père et sa mère l'avaient en aversion et n'avaient aucun plaisir à le voir; il était jaloux de son frère â̂né et désirait sans cesse lui nuire et lui faire obstacle; il n'obéissait pas à son frère â̂né, mais au contraire il lui résistait et s'opposait à lui.

(Un jour,) le prince Chan-yeou, précédé et suivi de son cortège, accompagné de chanteurs et de musiciens et entouré d'une grande multitude, sortit de la ville et jeta les yeux autour de lui; il vit des laboureurs qui, en défrichant la terre, faisaient sortir des insectes; des corbeaux les piquaient aussitôt de leur bec et les avalaient; le prince vit de loin ce spectacle; il en fut ému et en ressentit de l'afliction; né et grandi au fond du palais, il u'avait jamais vu pareille chose. Il demanda à ceux qui l'entouraient: „A quelle occupation se livre-t-on là pour que des êtres s'entredétruisent?" Ceux qui l'entouraient lui répondirent: „O prince, ce qui fait que votre royaume existe, e'est le peuple; ce qui fait que le peuple existe, c'est le boire et le manger; ce qui fait qu'on a à boire et à manger, c'est qu'on laboure les champs et qu'on plante les cinq sortes de céréales, grâce à quoi la vie est conservée". Le prince-héritier réfléchit et dit: „C'est bien douloureux! c'est bien douloureux!"

Étant allé un peu plus loin, il vit des hommes et des femmes qui ensemble filaient et tissaient; allant et venant, ils s'agitaient jusqu'à l'épuisement: ils se harassaient de fatigue et se donnaient beaucoup de peine. Le prince demanda: „A quelle occupation se - livre-t-on là ?"' Ceux qui l'entouraient lui répondirent: „O prince, 
les hommes filent et tissent pour faire des vêtements, afin de cacher les parties honteuses du corps et de couvrir les cinq mernbres". Le prince dit: „Ce sont là aussi des peines qui sont nombreuses".

Étant allé encore plus loin, il vit des hommes qui mettaient à mort des bœufs, des chameaux et des chevaux et qui dépeçaient des pores et des moutons. Lee prince demanda: "Qui sont ces gens?" Ceux qui l'entouraient lui répondirent: „Ces gens tuent (des animaux) pour en vendre la chair afin de gagner leur vie, et d'avoir de quoi se vêtir et de quoi manger". Le prince sentit en un instant tous les poils de sa peau se hérisser et il dit: "Que cela est étrange; que cela est douloureux; ceux qui tuent ont un cœur qui n'est pas bon; que le fort fasse souffrir le faible et qu'on tue les êtres vivants pour nourrir d'autres êtres vivants, c'est accumuler des maux pour plusieurs kalpas".

Étant allé encore plus loin, il vit une multitude d'hommes qui capturaient au filet des oiseaux et prenaient à l'hameçon des poissons; ils étaient injustes et trompeurs envers des êtres innocents; les forts opprimaient les faibles. Le prince demanda: "Qui sont ces gens, et comment nomme-t-on leur occupation?" Ceux qui étaient à ses côtés lui répondirent: „, 0 prince, ils prennent au filet des oiseaux et capturent des poissons. C'est par de telles occupations qu'on se procure de quoi se vêtir et de quoi manger". Quand le prince eut entendu ces paroles, des larmes de compassion remplirent ses yeux; (songeant que) dans le monde la multitade des êtres accomplit tontes sortes d'actes mauvais et que les tourments de tout genre sont sans fin, il était pénétré d'aflliction et n'était plus heureux. Il fit done rebrousser chemin à son char et rentra au palais.

Le roi lui demanda: „O prince, pourquoi, au retour de votre promonade êtes-vous si afligé?"' Le prince raconta au roi son père 31 
toutes les choses dont il a été parlé plus haut. Quand le roi l'eut entendu, il lui dit: „Toutes les choses que vous m'avez exposées ne peuvent pas ne pas exister de tout temps; comment suffiraientelles à vous afliger?" Le prince dit: „Je désire, ô roi, vous adresser un souhait. L'exaucerez-vous?” Le roi répliqua: „Vous êtes le seul fils que j'aime profondément; je ne m'opposerai point à ros intentions”. „Je désire, reprit le prince, avoir tout ce que ros trésors contiennent d'objets précieux, de boissons et d'aliments pour en faire universellement don". Le roi dit: „J'accorde ce que vous me demandez et je ne m'opposerai point à vos intentions".

Le prince Chan-yeou invita alors les ministres qui étaient à ses côtés à ouvrir les trésors du roi, à charger les objets précieux sur cinq cents grands éléphants et à sortir hors des quatre portes de la ville; puis il publia dans le royaume que ceux qui désiraient avoir des vêtements et des aliments vinssent prendre tout ce qu'ils désireraient; la voix du prince Chan-yeou fut entendue au loin dans les huit directions; tous les habitants se rassemblèrent comme des nuages, et, en peu de temps, on eut employé un tiers (de tout l'avoir du roi). Alors les ministres préposés à la garde des trésors vinrent dire au roi: „Le prince a déjà dépensé un tiers de ce qui était contenu dans les trésors; ô roi, il vous faut y réfléchir". Le roi répondit: „Celui qui fait cela est le prince; je ne saurais m'y opposer". Peu de temps après, les ministres considérèrent que ce qui fajsait exister le royaume, c'étaient les trésors, et que, quand les trésors seraient épuisés, le royaume aussi n'existerait plus que de nom; ils vinrent derechef dire au roi: „De tout ce que vous aviez d'objets précieux, les deux tiers sont dépensés; ô roi, il vous faut y réfléchir”. Le roi répondit: „Celui qui fait cela est le prince; je ne saurais m'y opposer. Cependant je vous autorise à aser de moyens 
dilatoires sans qu'aucun de vous déclare ouvertement ses sentiments". Quand le prince Chan-yeou voulut ouvrir les trésors, les ministres gardiens du trésor étaient absents parce qu'ils étaient partis; le prince les rechercha avec diligence, mais il les manqua et ne put les rencontrer. Le prince Chan-yeou dit: „Comment ces hommes de peu oseraient-ils s'opposer à mes volontés? Ce doit être le roi mon père qui leur a donné des instructions; or un fils doué de piété filiale ne doit pas épuiser entièrement les trésors de son père et de sa mère. Il faut maintenant que je cherche moi-même des richesses pour les distribuer à la multitude des êtres; si je ne suis pas capable de fournir en suffisance à la multitude des êtres vivants de quoi se vêtir et de quoi se nourrir conformément à leurs désirs, comment pourrais-je être appelé le prince fils du grand roi?"

Il rassembla alors les ministres et les fonctionnaires de tout rang et leur demanda conseil en disant: „Quelle est la condrite la meilleure à suivre pour celui qui cherche à se procurer des richesses?" Dans l'assemblée, le premier grand ministre répondit: „Dans ce monde, pour acquérir des richesses, le mieux est de labourer des champs; pour une semence on obtient dix mille fois plus". Un autre grand ministre dit: „Dans le monde, pour acquérir des richesses, le mî̌eux est de faire de l'élevage; quand on garde les troupeaux et que ceuxci se multiplient, l'avantage qu'on en retire est le plus considérable". Un autre grand ministre dit: „Dans le monde, pour acquérir des richesses, le mieux est d'aller sur mer pour recueillir de merveilleux joyaux; si on trouve la perle précieuse mo-ni (maṇi), on pourra donner en suffisance à tous les êtres conformément à leurs désirs". Le prince déclara: „Ce moyen-là est celui qui me plaît”.

Il entra alors dans le palais et dit au roi son père: „Moi, votre fils, je désire maintenant aller sur la grande mer pour y recueillir 
des joyaux merveilleux". Quand le roi eut entendu cette parole, il fut comme un homme qui s'étrangle et qui ne peut ni avaler ni cracher; il dit au prince: „Mon royaume vous appartient; tous les objets précieux qui sont dans mes trésors, prenez les et faites en usage à votre guise; pourquoi préeisément m'annoncer que vous vous proposez d'aller vous-même sur la grande mer? Vous êtes mon fils; vous êtes né et vous avez été élevé au fond du palais; quand vous dormiez, c'était au milieu des tentures; quand vous mangiez, vous aviez des aliments qui plaisent au goût; si maintenant vous allez au loin parcourir les routes, qui peut savoir la faim, la soif, le froid et le chaud (auxquels vous serez exposé)? En outre, dans la grande mer, la foule des périls est nombreuse: parfois il y a des démons malfaisants ou des dragons venimeux, des tempêtes ou des ouragans, des tourbillons ou des remous jaillissants, la montagne de la couleur de l'eau ${ }^{1}$ ) ou le grand poisson makara. Pour mille on cent mille qui partent, il en parvient (au but) un on deux. Comment se faitil que maintenant vous désiriez aller sur la grande mer? Je ne vous y autorise point". Le prince Chan-yeou se jeta alors de tout son long par terre; il étala ses deux mains et ses deux pieds et prononça cette parole: „Si mon père et ma mère ne m'autorisent pas à aller sur la grande mer, j'abandonnerai la vie ici-même et je ne me relèverai plus jamais". Quand le grand roi et ses épouses virent ce spectacle, leur yeux ne purent plus s'en détacher un seul instant; ils vinrent aussitôt exhorter le prince à se lever pour boire et pour manger. Le prince répliqua: „Si vous ne m'autorisez pas à aller sur la grande mer, je cesserai à jamais de boire et de manger".

1) La leçon 覀色之山, qui correspond à la leçon suvönglüg tar du manuscrit ouiggour, ne se trouve que dans l'édition du Tripitaka des Song; les autres éditions donnent la leçon 办泡之山 „la montagne d'écume d'eau”. 
Le roi et ses épouses furent saisis d'inquiétude et de chagrin; les assistants pleuraient, se chagrinaient et s'affigeaient; dans leur désolation, ils tombaient par terre à la renverse. Ainsi, pendant tout un jour, le prince-héritier ne but ni ne mangea; il en fut de même le second jour, puis le troisième jour et jusqu'au sixième jour; son père et sa mère, pleins d'inquiétude, craignirent qu'il ne pût pas se rétablir; le septième jour donc ils s'avancèrent et embrassèrent en criant ses mains et ses pieds; ils le réconfortèrent avec de douces paroles en l'invitant à se lever pour boire et pour mang'er : „Ce corps qui se nourrit, Iui disaient-ils, a besoin de boire et de manger pour se conserver intact; par la privation du boire et du manger, votre vie sera perdue”. Le prince-héritier répliqua: „0 mon père et ma mère, si vous ne consentez pas à ma demande, certainement je finirai mes jours ici sans jamais me relever'.

La première épouse dit alors au roi: „La résolution de votre fils ne saurait être abattue et il ne faut pas la repousser avec violence. Comment pourriez-vous supporter de voir ce fils perdre la vie en ce lieu? Je souhaite, ô grand roi, que rous répandiez sur lui votre bienveillance et que vous l'autorisiez à aller sur la grande mer, car, dans ce cas, il reste encore une chance sur dix mille, tandis que, si vous ne lui donnez pas votre autorisation, il mourra certainement en ce lieu".

Le roi donc renonça à faire opposition et accorda son consentement. Aussitôt le prince héritier Chan-yeou se leva tout joyeux et posa son visage sur les pieds du roi son père en signe de respect. Les femmes de l'escorte royale, ainsi que les belles jeunes filles, par centaines, par milliers et par myriades, se demandaient les unes aux autres: „Le prince-héritier Chan-yeou est-il maintenant mort ou vivant?" On leur répondit: „Présentement le prince-héritier s'est levé tout joyeux et il a bu et mangé". 
Le roi demanda au prince-héritier: „Vous avez voulu avec obstination aller sur la grande mer: que vous proposez-vous d'y faire?" Il répondit: „0 grand roi, je désire y recueillir les grands joyaux maṇi en quantité suffisante pour satisfaire aux besoins de tous les êtres vivants". Le grand roi alors promulgua une ordonnance disant: „Qui désire aller sur la mer? Ceux qui pourront faire le voyage d'aller et de retour auront des vêtements et de la nourriture pour sept générations et les joyaux précieux ne leur manqueront pas. Je fournirai le bateau nécessaire pour la route; le prince-héritier Chan-yeou désire lui aussi aller sur mer afin d'amasser les merveilleuses perles précieuses maṇi". Quand la foule apprit cette nouvelle, elle fut joyeuse et il y eut cinq cents hommes qui se rassemblèrent; ils dirent tous au grand roi: „Nous, maintenant, nous suivrons le prince-héritier".

En ce temps, dans le royaum de Po-lo-nai (Vârânạaî, Bénarès), il y avait un pilote qui, à plusieurs reprises, était allé sur la grande mer; il connaissait fort bien à quelles marques on distinguait sur la route les passages praticables et les obstacles; mais il était âgé de quatre-vingts ans et il était aveugle des deux yeux. Le grand roi de Po-lo-nai (Vârn̂nasî́, Bénarès) se rendit alors à la demeure du pilote et lui tint ce langage: „O pilote, l'unique fils ') que j'ai n'est point encore sorti en dehors de sa famille; veuillez condescendre à aller sur la grande mer; je souhaiterais qu'il fût à votre suite". Le pilote se mit aussitôt à pousser des cris et à se lamenter, disant: „O grand roi, les obstacles et les peines qu'on rencontre sur la grande mer ne sont pas en petit nombre; ceux qui partent se comptent par milliers et par myriades, mais il n'y en a qu'un ou deux

1) A ce propos, M. Pelliot (p. 24l, n. 1) remarque: „Le roi ne tient pas compte de son second fils, qu'il déteste". 
qui atteignent le but; ô grand roi, présentement comment se fait-il que vous ayez pu inviter le prince-héritier à parcourir au loin le chemin périlleux?" Le roi répondit au pilote: „C'est par compassion que je lui ai accordé mon consentement". Le pilote répliqua: „, $\mathrm{J}_{\theta}$ n'oserais contrevenir à votre ordre".

Pendant ce temps, le prince-héritier Chan-yeou avait équipé toute sa troupe de cinq cents hommes et les avait transportés sur le rivage de la grande mer. Or son frère cadet, le prince Ngo-yeou, fit cette réflexion: „Le prince-héritier Chan-yeou a toujours été chéri avec partialité par notre père et notre mère. Maintant il va s'embarquer sur la grande mer pour recueillir de merveilleux joyaux. S'il arrive au but et revient, notre père et notre mère m'abandonneront". Afant ainsi réfléchi, il alla annoncer à son père et à sa mère qu'il voulait lui aussi suivre maintenant le prince-héritier et aller sur la grande mer pour recueillir de merveilleux joyaux. Comme son père et sa mère lui demandaient les raisons de cette décision, il leur répondit: „Mon désir de le suivre vient de ce que, quand des circonstances périlleuses se présenteront au cours du voyage, nous pourrons mutuellement nous entr'aider comme des frères".

Lorsqu'on fut arrivé au bord de la grande mer, le bateau avait été amarré au moyen de sept câbles de fer; on séjourna là sept jours, et, chaque jour, au lever du soleil, le prince-héritier Chan-yeou frappait le tambour et faisait cette proclamation: „Qui parmi vous veut aller sur la grande mer? Que ceux qui veulent aller gardent le silence; mais s'il en est qui sont attachés de cœur à leur père et à leur mère, ou à leurs frères, ou à leurs femmes et à leurs enfants, ou aux joies du Jambudvîpa, qu'ils s'en retournent dès maintenant sans se préoccuper de moi. Pourquoi cela? Sur la grande mer, les périls ne sont pas en petit nombre; ceux qui partent se 
comptent par milliers et par myriades, mais il n'y en a qu'un ou deux d'entre eux qui parviennent au but". Après que cette proclamation avait été faite, comme l'assemblée gardait le silence, le prince coupait aussitôt un des câbles et le posait sur le bateau. Ainsi, chaque jour il faisait cette proclamation, et, lorsqu'arriva le septième jour, il coupa le septième câble et le mit sur le bateau. On hissa les voiles suivant la direction du vent. Grâce à la vertu bienheureuse du cœur affectueux du prince-héritier, on put parvenir sans aucun obstacle aux îles de la mer; quand on arriva à la montagne des joyaux précieux et qu'on atteignit au lieu où étaient les joyaux, le prince-héritier Chan-yeou frappa le tambour et fit cette proclamation: "Vous tous, sachez que la route pour venir ici est fort longue; hâtez-vous de rassembler des joyaux précieux". Après qu'on fut arrivé au terme d'une balte de sept jours, le prince tint de nouveau ce langage: „Ces joyaux sont de fort grand prix; dans tout le Jambudvipa il n'y a rien qui les vaille. Que personne de vous n'en emporte en trop grande quantité, car le bateau coulerait et vous n'arriveriez pas au terme du voyage. Que personne de vous n'en prenne en trop petite quantité, car la route pour venir ici est fort longue et vous ne seriez pas payés de vos peines". Quand l'embarquement eut été fini, il se sépara de ces hommes en leur disant: "Vous autres maintenant, faites un voyage de retour excellent et tranquille. Pour moi, j'irai plus avant pour rechercher les perles précieuses maṇi".

Alors donc le prince-héritier Chan-yeou, se mit en route avec le pilote aveugle. Après une semaine de marche, l'eau leur arriva jusqu'aux genoux; ils continuèrent à avancer, et, au bout d'une autre semaine, l'eau leur arriva jusqu'au cou; ils avancèrent encore pendant une semaine et c'est en nageant qu'ils purent passer. Quand 
ils furent arrivés au rivage de la mer, le sable en ce lieu était entièrement fait d'argent blanc; le pilote demanda: „Qu'y a-t-il en ce lieu?” Le prince-héritier lui répondit: „En ce lieu, il n'y a que du sable d'argent blane”. Le pilote reprit: „Quelque part dans l'une des quatre directions il doit $\mathrm{y}$ avoir une montagne d'argent; la voyez-vous?” Le prince-héritier dit: „Vers le Sud-Est une montagne d'argent est visible”. "Notre chemin, dit le pilote, passe au pied de cette montagne". Quand on fut arrivé à cette montagne, le pilote dit: „Ensuite on doit atteindre le sable d'or". Sur ces entrefaites, le pilote épuisé de fatigue perdit connaissance et tomba étendu sur le sol. Il dit ensuite au prince-héritier: „Ma vie ne peut plus durer longtemps et je vais mourir ici. 0 prince, vous marcherez vers l'Est pendant une semaine et vous devrez trouver une montagne d'or; à partir de cette montagne, continuant à marcher pendant une semaine, vous arriverez à un endroit où il n'y a rien que des lotus bleus; après une nouvelle semaine de marche, ce sera un lieu où il n'y aura rien que des lotus rouges. Après que vous aurez dépassé cette région des lotus, vous devrez trouver une ville faite avec les sept joyaux; c'est uniquement de l'or jaune qui constitue le rempart ${ }^{1}$ ); les tours $\mathrm{y}$ sont d'argent blanc et les créneaux ${ }^{2}$ ) de corail rouge; le $\operatorname{tch}^{c} \ddot{o}-k^{c} i u^{3}$ ) et le ma-nao (agate) y sont distribués de place en place; des perles vraies forment un réseau qui recouvre la ville; les septuples fossés ${ }^{4}$ ) y sont en lieou-li (vaidûrya) violet. C'est la

1) 却敵, littéralement „ce qui repousse les ennemis".

2)障板, littéralement „plaques qui font obstacle”.

3) 硨砸.

4) 浭壘, littéralement „les fossés et les talus”, mais il semble que le lieou-li violet tienne ici lieu de l'eau qui est dans les fossés. Plus loin, d'aillears, il n'est plus question que des 旉. 
résidence du grand roi-dragon de la mer; à l'oreille de ce roi-dragon il $\mathrm{y}$ a une perle précieuse maṇi qui fait se réaliser les désirs. Allez la lui demander. Si vous obtenez cette perle, vous pourrez avoir à profusion dans le Jambudrîpa les sept joyaux en plnie abondante, des vêtements ainsi que des boissons et des aliments, des remèdes pour toutes les maladies, des musiciens et des chanteurs; quoi que vous vouliez, vous n'aurez qu'à le dire; tout ce dont peuvent avoir besoin les êtres vivants quels qu'ils soient, vous le ferez tomber en pluie à votre volonté; c'est pourquoi on appelle ce joyau la perle précieuse qui fait se réaliser les désirs. $O$ prince, si vous obtenez cette perle, vous pourrez donner satisfaction à votre vœu primitif". Après que le pilote eut prononcé ces paroles, son souffle s'interrompit et sa vie prit fin. Alors le prince-héritier vint prendre dans ses bras le pilote; il cria et se lamenta, disant: „Comment se fait-il que notre destinée soit si frêle? j'ai perdu celui qui me tenait lieu de ciel" '). Alors il recourrit de sable d'or le pilote et l'enterra en ce lieu; il fit sept fois le tour (de la sépulture) en la tenant à sa droite, puis, après s'être prosterné, il partit.

Allant plus avant, il atteignit la montagne d'or. Après l'avoir dépassée, il vit des lotus bleus qui couvraient une place; sous ces lotus étaient des serpents venimeux bleus; ces serpents avaient trois sortes de venin, celui de leur morsure, celui de leur contact, celui de leur haleine; ces serpents venimeux se tenaient enroulés à la tige des lotus; ils regardaient le prince-héritier en ouvrant tout grands leurs yeux et en souffant. Alors le prince-héritier Chan-yeou entra dans le samûdhi du coeur bienveillant et, grâce à la force de ce samâdhi, quand il se mit en route, il passa en marchant sur

1) Cette métaphore désigne le plus sonvent le père par rapport au fils, ou le mari par rapport à la femme. 
les lotus ${ }^{1}$ ); alors les serpents venimeux ne lui firent ancun mal.

Par la force de son ccur bienveillant, il arriva tout droit à la résidence du roi-dragon. Sur les quatre faces du rempart, il y avait sept rangs de fossés; l'intervalle entre le rempart et les fossés était plein de dragons venimeux qui entrelaçaient leurs corps les uns aux autres, et qui, dressant leurs têtes et entrecroisant leurs cous, gardaient la porte de la ville. Quand le prince-héritier fut arrivé en dehors de la porte de la ville, il aperçut les dragons venimeux; son cœur bienveillant songea aussitôt à tous les êtres vivants da Jambudvîpa: „Maintenant, pensa-t-il, si mon corps que voici est détruit par ces dragons venimeux, vous, multitude de tous les êtres vivants, vous serez privés d'un grand avantage". Puis le princehéritier leva la main droite et dit aux dragons venimeux: „Sachez que c'est pour le bénéfice de tous les êtres vivants que je désiro voir maintenant le roi-dragon". Alors les dragons venimeux laissèrent le chemin libre et le prince-héritier put passer.

Après ${ }^{2}$ ) être ainsi parvenu jusqu'aux sept rangs de fossés et aux dragons venimeux qui gardaient le rempart, il arriva au pied de la porte du rempart. Il vit alors deux belles femmes qui filaient des fils de p'o-li (cristal) ${ }^{3}$ ). Le prince-héritier leur demanda : „Qui êtes-rous?” Elles répondirent: „Nous sommes les servantes préposées à la garde de la porte extérieure du roi-dragon". Après s'être ainsi informé, le prince entra plus avant et arriva au pied de la porte du milieu: il vit alors quatre belles femmes qui filaient des fils d'argent blanc; le prince-héritier leur demanda derechef: „Êtes-vous

1) Sur les feuilles de lotus, dit seule l'édition de Corée. Le miracle est que le prince marche soit sur les fleurs, soit sur les feuilles, sans qu'elles se conrbent.

2) $\nearrow_{7}$ n'est guère explicable, puisqu'il s'agit d'une chose passée par rapport à celle dont il va être maintenant question.

3) 頗 委. Le terte ouigour dit simplement „un fil précieur". 
des épouses du roi-dragon?" Elles répondirent: „Non; nous sommes les servantes préposées à la garde de la porte du milieu". Après s'être ainsi informé, le prince entra plus avant et arriva jusqu'à la porte intérieure; il vit alors huit belles femmes qui filaient des fils d'or jaune; le prince leur demanda: „Qui êtes-vous?” Elles répondirent: „Nous sommes les servantes, préposées à la garde de la porte intérieure du roi-dragon". Le prince-héritier leur dit: „Annoncez de ma part au grand roi-dragon de la mer, que le prince-héritier Chanyeou, fils du roi de Po-lo-nai (Vârânaasî, Bénarès) dans le Jambudvîpa) est venu exprès pour le voir et maintenant est à la porte".

Quand les préposées à la garde de la porte eurent fait cette annonce, le roi-dragon, en entendant ce qu'elles disaient, se demanda avec surprise comment cela avait pu se produire; il fit cette réflexion: „Si cet homme n'était pas un homme pur et excellent dont la vertu porte bonheur, il n'aurait pas trouvé le moyen de venir de loin en parcourant un chemin si périlleux". Aussitôt il l'invita à entrer dans son palais. Le roi-dragon sortit done pour lui faire accueil.

Dans le palais de ce roi-dragon, le sol était constitué par du lieou-li (vaị̣̂urya) violet; les couches et les sièges étaient faits avec les sept joyaux; c'étaient des scintillements multipliés qui éblouissaient la vue. Le roi-dragon le pria de s'asseoir et ils se demandèrent l'un à l'autre de leurs nouvelles. Le prince-héritier Chan-yeou en profita pour lui expliquer la Loi et pour lui enseigner la profitable joie ${ }^{1}$ ); il lui donna toutes sortes d'instructions et lui exposa ce qui concerne la libéralité et ce qui concerne les défenses et ce qui concerne les devas et les hommes. En ce temps, le grand roi-

J) C'est-à-dire, comme l'explique le texte onigour, qu',,il lui enseigna le profit de donner des aumônes d'un ceur grandement joyeux". 
dragon de la mer fut très joyeux dans son cœur et lui demanda: „Si, venant de loin, vous avez pris de la peine pour parcourir cette route, quel objet vous fallait-il obtenir?"' Le prince-héritier répondit: „O grand roi, dans tout le Jambudvîpa les êtres vivants endurent des soufrances illimitées à cause des vêtements, des richesses, du boire et du manger. Maintenant je voudrais obtenir la perle précieuse qui est à votre oreille gauche, la perle maṇi qui fait se réaliser les désirs". Le roi-dragon lui répondit: „Acceptez que je subvienne quelque peu à votre entretien pendant une semaine et onsuite je vous la donnerai en offrande". Alors le prince-héritier Chan-yeou accepta la requête du roi-dragon et, quand la semaine fut écoulée, il obtint la perle précieuse maṇi et retourna dans le Jambudvîpa. A ce moment, le grand roi-dragon de la mer chargea la multitude des dieux-dragons de l'accompagner en volant dans les airs.

(Le prince Chan-yeou) parvint ainsi à ce rivage (qu'il avait autrefois quitté) et vit son frère cadet Ngo-yeou. Il lui demanda: „Où sont maintenant vos compagnons et camarades?". Il répondit: „O Chan-yeou, le bateau s'est englouti dans les eaux et tous ont péri; moi seul, votre frère cadet, en m'accrochant à un cadavre ${ }^{1}$ ), j'ai pu sauver ma vie; mais toutes mes richesses sont entièrement perdues". Chan-yeou lui répondit: „Dans le monde, un homme n'a rien de plus précieux que sa propre personne”. „Non, répliqua le frère cadet; l'homme aimerait mieux mourir riche que de vivre pauvre. Si je sais qu'il en est ainsi, c'est que, étant allé dans un cimetière, je l'ai entendu dire aux mânes des morts". Le princehéritier Chan-yeou était sincère et droit de nature; il révéla donc la vérité à son frère cadet en lui disant: „Si vous avez perdu vos

1) 死 Р. Le mot ture correspondant sïuqün est obseur; M. Huart l'a traduit par „amarre"; M. Pelliot par „épave". 
joyaux, cela n'a pas d'importance, car j'ai obtenu la perle précieuse du roi-dragon, la perle mani qui fait se réaliser les désirs". Son frère cadet lui demanda: „Maintenant où se trouve-t-elle?" Chanyeou lui répondit: „Elle est maintenant dans mon chignon”. Quand son frère cadet eut entendu cette parole, son cœur en conçut de l'envie; irrité et chagrin, il fit cette réflexion: „Mon père et ma mère ${ }^{1}$ ) ont toujours chéri (Chan-yeou) avec partialité; maintenant en outre il a obtenu la perle précieuse maṇi; dorénavant ma personne sera méprisée par mon père et par ma mère plus encore qu'un tesson de poterie". Après avoir fait cette réflexion, il dit à Chanyeou: „Quel bonheur et quelle chose excellente que vous ayez obtenu cette perle précieuse; maintenant dans ce royage périlleux, il faut que nous la gardions bien". Alors Chan yeou détacha la perle précieuse et la remit à son frère cadet Ngo-yeou en lui faisant cette recommandation: "Quand vous serez fatigué et que vous vous coucherez pour dormir, c'est moi qui la garderai. Quand je me coucherai pour dormir, c'est vous qui devrez la garder. En ce moment, e'est votre tour, Ngo.yeou, de la garder".

Quand son frère aîné fut endormi, (Ngo-yeou) se leva et chercha deux broches de bambou sec avec lesquelles il creva les denx yeux de son frère aîné, puis il partit en emportant la perle.

Or Chan-yeou se mit à appeler son frère cadet Ngo-yeou en lui disant: „Il y a un brigand qui m'a crevé les deux yeux et qui est parti en emportant la perle précieuse". Comme Ngo-yeou ne répondait pas, son frère aîné se dit avec inquiétude: „Mon frère cadet Ngo-yeou paraît avoir été tué par les brigands". Criant ainsi a haute voix, ses accents émouvaient les dieux; pendant longtemps

1) Quoique, d'après le début du récit, la mère de $\mathrm{Ngo-yeou} \mathrm{soit} \mathrm{distincte} \mathrm{de} \mathrm{la} \mathrm{mère}$ de Chan-yeon, ces deux femmes n'en font plus qu'ane ici. 
rien ve lui répondit, mais alors le dieu d'un arbre prononça cette parole: „C'est votre frère cadet Ngo-yeou qui est votre méchant brigand; il a crevé vos deux yeux et est parti en emportant la perle précieuse. A quoi sert maintenant d'appeler Ngo-yeou?" Quand le prince-héritier Chan-yeou eut entendu cette parole, avec chagrin il contint son ressentiment; il s'afligea et fut tourmenté.

Cependant Ngo-yeou, emportant avec lui la perle précieuse, était revenu dans son pays; il eut une entrevue avec son père et sa mère et leur dit: „O mon père et ma mère, grâce à ma vertu qui porte bonheur, j'ai pu revenir sain et sauf. Quant au prince-héritier Chan-yeou et à tous ses compagnons, ils avaient trop peu de cette vertu qui porte bonheur et e'est pourquoi tous sont morts noyés". Quand son père et sa mère entendirent cette parole, ils se lamentèrent $\grave{a}$ grands cris; ils s'évanouirent de chagrin et tombèrent étendus à terre. On leur aspergea le visage d'eau frâ̂che et, au bout de quelque temps, ils revinrent à eux. Le père et la mère dirent à Ngo-yeou: „Comment se fait-il que vous ayez pu revenir ${ }^{1}$ ) en rapportant ceci?" 2) Quand Ngo-yeou eut entendu cette parole, il en conçut de l'inquiétude et il enterra la perle précieuse dans le sol. .

En ce temps, le prince héritier Chan-yeou avait eu les deux yeux crevés; les broches de bambou sec n'ayant été enlevées par personne, il errait en tournant de ci et de là et nul ne savait où il était allé. Il était alors accablé de peine et souffrait beaucoup de la faim et de la soif; soit qu'il cherchât à vivre, soit qu'il cherchât à mourir ${ }^{3}$ ), il n'y pouvait parvenir. Graduellement il avança et arriva au royaume

1) Dans l'édition de Corée le mot 回 est remplacé par 面 qui n'offre pas de sens.

2) Ils ont sans doute en vue la perle.

3) Il restait dans un état intermédiaire entre la vie et la mort. 
de Li-che-pa 利師践. Le roi de Li-che-pa avait une fille qu'il avait autrefois promise en mariage au prince-héritier Chan-yeou, fils du roi de Po-lo-nai (Varânasî̀, Bénarès). Or le roi de Li-che pa avait un bouvier nommé Lieou-tcheng 留烝 qui menait cinq cents bœufs dans des pâturages pour le roi de Li-che-pa. Commele prince-béritier Chan-yeon était assis au bord de la route, le troupeau des bœufs le renversa et le foula aux pieds; mais il y avait parmi eux un roi des bœufs qui se tint au-dessus du prince-héritier les quatre pieds écartés et il invita la foule des bœufs à défiler; quand ils eurent tous passé, il déplaça ses pieds, et, décrivant un cercle en le laissant à sa droite, il tourna autour de lui; retournant la tête en arrière, il sortit sa langue et lécha les deux yeux du prince-héritier; il enleva ainsi les deux broches de bambou. C'est alors que le bouvier, en cherchant (son bœuf), aperçut (le prince-héritier); il lui demanda qui il était. Chan-yeou se dit à part lui : „Maintenant je ne dois pas raconter mon histoire; car si je révèle ce qui est arrivé, je serai cause que mon frère cadet subira de grands tourments". Il répondit donc: „Je suis un mendiant aveugle". Le bouvier, le considérant dans toute sa personne, s'aperçut qu'il avait des caractéristiques remarquables; il lui dit: „Ma maison est proche; je vous y entretiendrai". Le bouvier prit donc avec lui Chan-yeou et le ramena dans sa demeure; il lui donna toutes sortes de boissons et d'aliments; il enjoignit à toutes les personnes de sa maison, hommes ou femmes, grands ou petits, de servir cet homme comme lui-même. Au bout d'un mois et quelques jours, les gens de la famille se lassèrent et tinrent ce propos: "Les ressources de notre famille ne sont pas abondantes; comment pourrions-nous toujours nourrir cet aveugle?" Quand Chan-yeou eut entendu cette parole, son cceur en fut désolé; lorsque cette nuit fut écoulée et que vint le matin, il dit à son 
hôte: „Maintenant je veux partir". Son hôte lui répondit: „Y a-t-il quelque chose qui vous ait déplu pour que vous vouliez nous quitter?" Chan-yeou répondit: „La situation qui est réglée par les rapports du visiteur et de l'hôte ne doit pas durer longtemps". Il ajouta: „Si vous arez quelque affection à mon égard, faites pour moi une guitare ${ }^{1}$ ) sonore et menez-moi dans un endroit où il y ait beaucoup d'hommes rassemblés, dans quelque agglomération de grande ville. Alors son hôte lui fournit ce qu'il désirait et le mena dans la ville de Li-che-pa, en un endroit où il y avait beaucoup d'hommes rassemblés, puis il s'en retourna après l'avoir bien installé.

Chan-yeou était fort habile à jouer de la guitare; sa musique était harmonieuse et plaisait au cœur de la multitude; tous les gens de la foule lui apportaient à boire et à manger en telle abondance que cinq cents mendiants qui étaient sur la ronte de Li-che-pa purent tous être rassasiés.

Or le roi de ce pays avait un verger qui était fort prospère mais qui était constamment ravagé par les corbeaux et les moineaux. Le surveillant de ce verger dit à Chan-yeou: „Si vous me protégez contre ces corbeaux et ces moineaux, je vous soignerai bien". Chan-yeou répondit: „Je n'ai plus mes deux yeux; comment pourrais-je me charger de chasser pour vous les corbeaux et les moineaux?" Le gardien du verger lui dit: „J'ai un moyen; j'attacherai une corde au sommet de tous les arbres et j'y adapterai des clochettes de cuivre; vous serez assis au pied des arbres, et, quand vous entendrez le cri des corbeaux et des moineaux, vous tirerez le bout de la corde". Chan-yeou répondit: „S'il en est ainsi, je puis me rendre au pied des arbres". Quand le gardien l'eut bien installé là, il le quitta et s'en

1) 箑. 
alla. Chan-yeou protégea done (les fruits) contre les corbeaux et les moineaux, et en même temps, il jouait de la guitare pour se délecter.

Sur ces entrefaites, la fille du roi de Li-che-pa, accompagnée de son escorte, vint se promener dans le verger; elle aperçut cet aveugle, se rendit auprès de lui et lui demanda qui il était. „Je suis, réponditil, un mendiant aveugle". Quand la fille du roi l'eut vu, son cœur conçut des pensées d'amour et elle ne put plus s'éloigner de lui. Le roi, de son côté, envoya des gens appeler sa fille, mais elle refusa de partir et demanda qu'on lui envoyât à manger. Quand elle eut fini de boire et de manger avec cet aveugle, elle dit au grand roi: „O roi, si maintenant vous me prenez pour me donner à cet aveugle, cela comblera mes voeux”. Le roi répliqua: „Yous étes possédée par quelque esprit malfaisant qui vous a affolée en sorte que votre esprit est troublé. Comment demeureriez-vous en compagnie de cet aveugle? ne savez-vous pas que votre père et votre mère vous ont précédemment promise en mariage au prince-héritier Chan-yeou, fils du roi de Po-lo-nai (Vârânạasi, Bénarès)? Maintenant Chan-yeou est allé sur la mer et n'est point encore revenu. Comment pourriez-vous devenir la femme de ce mendiant?" La fille répondit: „Même si je devais en perdre la vie, jamais je ne me séparerai de lui". Quand le roi eut entendu ces paroles, il ne put maintenir son opposition: il envoya done des gens amener cet aveugle et l'installer dans une chambre de repos.

Alors la fille du roi se rendit auprès de l'aveugle et lui dit: "Sachez que maintenant je veux que vous et moi soyons mari et femme”. Chan-yeou lui répondit: „De qui êtes-vous la fille pour vouloir être ma femme?" Elle répondit: „Je suis la fille du roi de Li-che-pa”. Chan-yeou répliqua: „Vous êtes la fille du roi et moi je suis un mendiant; comment pourriez-vous me témoigner du respect?" 
La femme dit: „Je vous servirai de tout mon cœur et je ne m'opposerai à aucune de vos volontés". Quand quatre-vingt dix jours se furent ainsi écoulés, cette femme s'absenta pour quelque menue occupation sans en avertir son mari; quand elle revint au bout de quelque temps, Chan-yeou lui adressa des reproches, disant: „Vous êtes sortie secrètement sans m'en avertir; d'où revenez-vous?" Elle répondit: „Je n'ai rien commis de caché”. „Qu'il s'agisse ou non de quelque chose de caché, reprit son mari, qui connaîtra ce que vous avez tait?" Sa femme affligée eut les yeux pleins de larmes; elle prononça alors cette imprécation: „Si j'ai commis quelque action cachée, puissent vos deux yeux ne jamais guérir; mais, s'il n'en est rien, puisse l'un de vos yeux redevenir comme il était auparavant". Dès qu'elle eut prononcé ce vœu, la pupille d'un des yeus de son mari remua et cet oil redevint sain comme auparavant; l'éclat de la prunelle fut brillant comme celui d'une étoile filante; la vue (du prince) fut claire et pénétrante et il put voir sa femme. Sa femme lui dit: „Eh bien, me croyez-vous?" Comme Chan-veou souriait, elle ajouta: „Vous ne connaissez pas ma bonté envers vous; je suis la fille du roi d'un grand royaume; vous êtes un homme de basse extraction; or je vous ai servi de tout mon cœur et vous n'avez pas pris confiance en moi". Son mari lui dit: „Savez-rous qui je suis?" Elle répondit: „Je le sais: vous êtes un mendiant”. „Non, reprit son mari, je suis le prince-héritier Chan-yeou, fils du roi de Po-lo-nai (Vârânạsî, Bénarès)". „Vous êtes un grand sot et un grand fou, répliqua sa femme; comment se fait-il que vous prononciez une telle parole? Le prince-héritier Chan-yeou, fils du roi de Po-lo-nai, est allé sur mer et n'est point encore revenu. Pourquoi prétendez-vous être lui? e'est là un mensonge". Chan-yeou dit: „Depuis ma naissance, je n'ai jamais menti". „Que ce soit vrai ou faux, répondit 
sa femme, qui le croira?" Son mari dit: „Si j'ai menti pour vous tromper, que mon autre œil ne soit jamais guéri; mais si j'ai dit vrai, que cet œil redevienne comme il était primitivement, et que vous puissiez en être témoin!" Quand il eut conçu cette pensée, son souhait se réalisa aussitôt; la lumière de sa prunelle scintilla et remua tout comme auparavant; les deux yeux de Chan-yeou étant guéris, son visage était admirable et il était un homme parfait; sa beauté était insurpassable et ne pouvait être comparée à aucune autre dans le monde.

Quand sa femme eut vu cela, elle en conçut de la joie dans son coour, comme si elle avait reçu la faveur d'un saint; elle le contemplait sur tout son corps et ses yeux ne pouvaient se détacher de lui. Elle entra aussitôt dans le palais et annonça au roi son père: „Maintenant celui qui est mon mari n'est autre que le prince-héritier Chan-yeou”. Le roi lui dit: „Vous êtes folle et vous avez l'esprit bouleversé; quelque démon malfaisant vous possède, pour que vous parliez ainsi. Le prince-héritier Chan-yeou est allé sur mer et n'est point encore revenu. Maintenant comment pouvez-vous donner le nom du prince-héritier à un mendiant?" Sa fille répliqua: „Il n'en est point ainsi. Si vous ne me croyez pas, allez le voir". Le roi alla donc le regarder, et, quand il l'eut considéré, il reconnut qu'il était bien le prince-héritier Chan-yeou; pénétré de crainte, il se dit que, si le roi de Po-lo-nai apprenait ce qui s'était passé, il aurait contre lui un ressentiment qui ne serait pas petit. Il vint donc s'excuser auprès du prince-héritier en lui disant que réellement il ne l'avait pas reconnu. Le prince-héritier lui dit: „N'en ayez aucun chagrin; faites de ma part des dons à ce gardien de boufs". Le roi de Li-che-pa donna aussitôt de l'or, de l'argent, des joyaux, des vêtements, et des aliments à celui qui menait paître les cinq cents 
boufs; cet homme tout joyeux exprima des remercîments infinis en disant: „Le prince-héritier Chan-yeou n'a reçu de moi que des bienfaits infimes; or voici de quelles richesses il me récompense!" Alors ce gardien de bœufs proclama à haute voix au milieu de la nombreuse assemblée: „Pour les libéralités cachées il y a des récompenses manifestes; pour les actes de libéralité la récompense est immense". Alors les cœurs de ceux qui constituaient la grande assemblée éprouvèrent de la joie; tous conçurent des sentiments de libéralité pour venir au secours de tous les êtres, et firent de la recherche du Buddha leur occupation principale; les dieux qui étaient dans les airs louèrent ces hommes et prononcèrent des paroles d'approbation.

Cependant le prince-héritier Chan-yeou, au temps où il n'était pas encore allé sur la mer et où il résidait dans le palais, entretenait une oie sauvage blanche; qu'il s'habillât ou qu'il mangeât, qu'il marchât ou qu'il fût immobile, qu'il fût assis ou qu'il fût couché, il l'avait toujours avec lui. Alors donc l'épouse (du roi de Po-lo-nai) se rendit auprès de cette oie sauvage et lui dit: , Au temps où le prince-héritier était ici, vous demeuriez toujours avec lui; maintenant il est allé sur la grande mer et n'est point encore revenu; on ne sait s'il est vivant ou s'il est mort et je ne parviens pas à savoir ce qu'il en est réellement. Comment maintenant no penseriez-vous pas avec émotion au prince-héritier?' Quand l'oie sauvage eut entendu ces paroles, elle se tordit en poussant des cris de douleur et les larmes remplirent ses yeux; elle répondit: „0 épouse du grand $\mathrm{roi}^{1}$ ), puisque vous désirez me charger de rechercher le prince-héritier, je ne saurais contrevenir à votre ordre". Alors

1) L'édition de Corée est seule à donner le mot duire: „ố principale épouse". 
l'épouse écrivit de sa propre main une lettre qu'elle attacha au cou de l'oie sauvage.

Cette oie sauvage avait autrefois demandé au prince-héritier où se trouvait la grande mer; elle s'éleva donc dans les airs, tourna en volant et partit. Quand l'épouse la vit, son cœur en conçut du réconfort: „Maintenant, pensa-t-elle, cette oie saurage certainement obtiendra des informations sûres qui m'apprendront si mon fils est mort ou vivant". L'oie sauvage arriva en volant jusqu'à la grande mer; elle la traversa et la parcourut en tous sens, mais, malgré ses recherches, elle ne put rien voir; progressivement, elle atteignit le royaume de Li-che-pa; elle aperçut de loin le prince-héritier Chanyeou qui se tenait devant le palais; cette oie sauvage, laissant tomber son corps et repliant ses ailes, se rendit auprès de lui; quand elle fut arrivée, elle se réjouit en poussant des cris plaintifs. Le prince-héritier prit alors la lettre de sa mère; il lui rendit hommage, en la mettant au sommet de sa tête, puis il rompit le cachet et la lut. Il apprit ainsi que son père et sa mère nuit et jour se lamentaient, ne faisant que songer au prince-héritier, et que leurs deux yeux en avaient perdu la vue. Le prince-héritier écrivit aussitôt lui-même une lettre dans laquelle il exposait à son père et à sa mère tout ce que nous arons raconté plus haut, et, à son tour, il attacha cette lettre au cou de l'oie sauvage. Celle-ci, toute heureuse, s'en revint à Po-lo-nai.

Quand le père et la mère furent en possession de la lettre du prince-héritier, ils bondirent de joie et se répandirent en actions de grâce infinies. Comme ils avaient ainsi appris que le prince-héritier avait été mis en péril et maltraité par son frère cadet Ngo-yeou, qui lui avait enlevé la perle précieuse et lui avait infligé des tourments, le père et la mère firent aussitôt mettre des entraves aux 
pieds et aux mains de Ngo-yeou; ils lui serrèrent le cou dans une chaîne et l'enfermèrent en prison. Puis ils envoyèrent un messager dire au roi de Li-che-pa: „Pourquoi maintenant retenez-vous le prince-héritier, ce qui est cause que nous nous affligeons". Quand le roi de Li-che-pa eut entendu cette parole, son cœur en conçut de la crainte; aussitôt il fit revêtir d'un costume magnifique le prince-héritier pour le reconduire jusque dans son pays. Le princehéritier envoya un messager annoncer au roi de Li-che-pa: "Chanyeou est revenu de son voyage sur la grande mer ${ }^{1}$ )". Alors le roi de Li-che-pa disposa des chanteurs et des musiciens pour former un cortège par devant et par derrière; il balaya et arrosa le sol et brûla des parfums; il suspendit des oriflammes et des dais en soie; frappant des cloches et faisant résonner des tambours, il alla au loin à la rencontre du prince-héritier; quand il l'eut ramené dans son palais, il le fiança à sa fille, puis il le renvoya avec une escorte dans le royaume de Po-lo-nai.

Qnand le père et la mère apprirent que le prince-héritier revenait, ils en eurent une joie sans limites; ils montèrent sur un grand éléphant illustre; des chanteurs et des musiciens se faisant entendre, le sol étant balayé et arrosé, des parfums étant brûlés, des oriflammes et des dais étant suspendus, ils allèrent au loin à la rencontre du prince-héritier; tous les habitants du royaume, tant les hommes que les femmes, apprenant que le prince-héritier, après être allé sur la grande mer, était revenu sain et sauf, se réjouirent infiniment et tous aussi sortirent pour aller à sa rencontre.

Le prince-héritier Chan-yeou s'avança et rendit hommage à son père et à sa mère en posant son visage sur leurs pieds; le roi et

1) Il feint d'arriver pour la première fois afin que l'aceueil qu'il reçoit soit digne de lui. 
sa femme avaient les yeux obscurcis et ne pouvaient voir l'extérieur du prince; ils le palpaient avec leurs mains en disant: „Etes-vous bien notre fils Chan-yeou? Voici à quel point ${ }^{1}$ ) nous, votre père et votre mère, nous avons été affligés en pensant à vous". Quand le prince-héritier eut fini de demander à son père et à sa mère des nouvelles de leur santé, il leva la main et, d'une voix forte, il remercia les divers rois des petits royaumes ainsi que toute la foule comprenant la multitude des fonctionnaires et la population du royaume; il leur dit: „Je vous remercie, ô foule nombreuse, pour la peine que vous avez prise: maintenant, retournez-vous en".

Le prince-héritier Chan-yeou dit au roi son père: „Où se trouve actuellement mon frère cadet Ngo-yeou?" Le roi lui répondit: „Il ne faut pas que vous vous informiez de lui; un si méchant homme est maintenant en prison et on ne doit pas le relâcher'. Le princehéritier Chan-yeou répliqua: ,Je désire que vous relâchiez Ngo-yeou pour que je puisse le voir." Quand il eut répété cette parole par trois fois, le roi ne voulut plus le contrarier et ourrit douc la porte de la prison. Alors Ngo-yeou, des entraves aux pieds et aux mains et une chaîne enserrant son cou, vint se présenter devant Chan-yeou; quand son frère aîné le vit dans cet état, il dit à son père et à sa mère: „Délivrez mon frère cadet de la chaîne qui l'enserre”. Quand on l'eut délivré de sa chaîne, il vint à lui et le prit dans ses bras; avec des bonnes paroles il lui donna ses instructions; il s'informa de lui en termes affables, et lui dit: „N'avez-vous pas enduré des peines très grandes? Ma perle précieuse que vous possédiez, où se trouve-t-elle maintenant?" Quand il eut répété cette question par trois fois, (Ngo-yeou) répondit enfin: „Elle est en tel lieu”.

1) Au point d'en perủre la vue. 
conte bouddhique de Kalyânạamkara et Pâpaṃkara.

Quand le prince-héritier Chan-yeou fut rentré en possession de sa perle précieuse, il alla auprès de son père et de sa mère, et, se prosternant à deux genoux, il brûla de merveilleux parfums de prix, puis il fit cette prière solennelle: „Si cette perle est vraiment le joyau qui fait se réaliser les désirs, puissent les deux yeux de mon père et de ma mère redevenir limpides comme auparavant". Dès qu'il eut formulé ce vœu, aussitôt (les yeux de ses parents) redevinrent comme auparavant et le père et la mère purent voir leur fils. Ils sautèrent de bonbeur et se réjouirent sans limites.

Puis, lorsque vint le matin du quinzième jour du mois, le prince héritier Chan-yeou, s'étant lui-même lavé et ayant revêtu des habits propres, brûla de merveilleux parfums de prix et monta sur une tour élevée; tenant en main le brûle-parfums, il rendit hommage à la perle précieuse mani avec son visage et arec le sommet de sa tête et fit ce vœu: "C'est pour le bien de tous les êtres vivants du Jambudivîpa que j'ai enduré de grandes souffrances afin d'obtenir cette perle merveilleuse". Aussitôt du côté de l'orient un grand vent s'éleva qui supprima de son souffle les nuages et les brouillards, en sorte que toute l'atmosphère fut pure d'une manière limpide; en même temps, tout ce qu'il y avait dans le Jambudvîpa de souillures, d'urine et d'excréments, de cendres et de broussailles, fut entièrement nettoyé par l'action du vent frais. Par la vertu éminente de la perle, sur toute l'étendue du Jambudvîpa il plut du riz en pleine maturité produit spontanément; il était parfumé et doux, moëlleux et fin; il était exquis en couleur et au goût; tous les fossés en furent pleins et il s'accumulait jusqu'à la hauteur des genoux. Ensuite il plut des vêtements excellents et des habits parfaits, des colliers de perles, des agrafes et des bracelets. Ensuite il plut de l'or, de l'argent, des substances précieuses des sept sortes et des harmonies merveilleuses 
500 ED O OARD OHAVANNS, Une version chinoise du etc.

en foule. Pour ne dire que l'essentiel, tout ce qui sert au contentement des êtres vivants quels qu'ils soient, se trouva fourni en abondance. Telle est la manière dont le bodhisattva, en exerçant sa grande compassion, et en pratiquant la dânapâramitâ, fournit aux êtres vivants quels qu'ils soient toutes les satisfactions.

Le Buddha dit à Ânanda: En ce temps, celui qui était le grand roi de Po-lo-nai, e'est présentement mon père Yue-t'eou-t'an (Çuddodhana); celle qui, en ce temps, était la mère, c'est présentement ma mère, l'épouse Mo-ye (Mâyâ); celui qui, en ce temps, était le prince royal Ngo-yeou, c'est maintenant T'i-po-ta-to (Devadatta); celui qui, en ce temps, était le prince royal Chan-yeou, n'est autre, aujourd'hui, que moi-mème. 